difficult. The name spinifex would, however, be especially suitable for plants of Triodia with their irritating prickly leaves like long sharp-pointed knitting needles bristling in all directions as the spines of a hedge-hog. S. paradoxus is not prickly and is sometimes spoken of as 'sandhills cane-grass'. Thus Sturt seems to have been the originator of the mistake and has been followed by most of the later explorers. How he came to make it is, however, still a puzzle.

I enclose two photographs (Figs. 1 and 2) taken on a recent camel trip to Ayers Rock, a very unget-atable place. These illustrate well the abundance and appearance of Triodia in parts of Central Australia.

University,

J. B. Cleland.

Adelaide, South Australia. Jan. 10.

Is reply to Prof. Cleland's letter, which has been referred to me by the Editor of NATuRE, I may say that I am quite cognisant of the use of the popular name 'spinifex' for most of the Australian species of Triodia, and am well aware of their dominance in the more arid regions of the interior. In referring to Spinifex paradoxus, however, I merely directed attention to an extremely interesting example of a xerophytic grass with rigid woody culms and male and female spikelets on different plants. The statement that Spinifex paradoxus is "one of the dominant species in the Sturt Desert of Australia" was not taken from the book under review, but was gleaned from the papers quoted below.

According to Prof. J. A. Prescott in his "Soils of Australia in relation to Vegetation and Climate" (Austral. Couneil Sei. Ind. Res. Bull, 52, p. 54 ; 1931), there are two extensive desert areas, consisting mostly of parallel sand-ridges bearing a desert grass association, the principal grasses being "spinifex" or "porcupine grass" (Triodia pungens and T. irritans), and "cane grass" (Spinifex paradoxus), the latter common in the more eastern desert-areas but the former more universal in their occurrence.

Prof. Cleland, himself, in a paper with J. M. Black and L. Reese on the flora of the north-east corner of South Australia (Trans. Roy. Soc. S. Austral., 49, $103-120 ; 1925)$, states (p. 106) that the vegetation on the more or less shifting sandhills is meagre but very characteristic. Among the most striking plants on the higher sandhills are Spinifex paradoxus and Triodia, etc.

Prof. Cleland, of course, is a well-known authority on the vegetation of these desert-areas, having made several expeditions through them, and his statement that Spinifex paradoxus is not one of the really dominant grasses should be accepted as conclusive.

THE Reviewer.

\section{A Unitary Model of the Galactic System}

However discordant the present views in astronomy are, they all concur in considering Milky Way clouds as real agglomerations of stars and regard the collection of stars surrounding the sun as one of these clouds ('local system'). To the commonsense evidence based on the apparently cloud-like character of the Milky Way one usually adds the following argument. The space density of stars in the direction of some galactic clouds first decreases and then increases again, thus indicating the existence of separate stellar agglomerations. The general belief in the existence of the 'local system' is based on two facts : the well-known decrease in apparent star density with increasing distance from the sun, and the distribution of brighter early stars and diffuse nebulæ along a circle inclined at several degrees to the galactic equator.

Recent discoveries have shown that our galactic system is quite comparable in size with a large extragalactic nebula and disposed of the comforting idea of transparent galactic space. They throw new light on this vexed problem, suggesting doubts as to the very existence of separate Milky Way clouds. Against the well-known arguments in favour of the existence of such clouds, we may now bring the following :

(1) Owing to the shearing effects of the galactic rotation, such galactic clouds would be dissipated in the course of a few revolutions, the period of which is considerably shorter than the shortest permissible cosmic time scale. Such a flattened system of clouds would be, moreover, very unstable even without shearing effects of rotation.

(2) A galaxy of such a complicated character would be quite an exceptional formation among extragalactic nebulæ, which never resemble an accumula. tion of separate clouds.

(3) It should be indicated that the abovementioned results of earlier star counts are not sub. stantiated by recent work by Schalén, who dealt with absolutely bright stars. In all the regions investigated from Cygnus to Auriga, no increase in density has been found as far as $3 \cdot 5$ kiloparsecs from the sun ; only in the region of Scutum $-20^{\circ}-30^{\circ}$ from the direction towards the galactic centre - do Schalén's curves indicate condensations of stars.

The arguments against the existence of a 'local system' can be summarised as follows:

(1) Not the slightest dynamical effects of this system has ever been found in stellar movements, which seem to be governed by the distant galactic centre in Sagittarius.

(2) An apparent arrangement of brighter $B$ stars largely depends upon a rich but isolated star group in Orion, without which the "secondary galaxy" would be lost in a general star field. The distribution of diffuse nebulæ showing continuous spectra simply repeats the distribution of dark nebulæ.

(3) The decrease in star density with the distance from the sun can be explained by cosmic absorption of $0 \cdot 3-0.4$ mag. per $10^{3}$ parsecs in visual light; this value being substantiated by independent evidence. Two diametrically opposite regions of obscuration cross the 'local system' : one in Ophiuchus (galactic long. $330^{\circ}-30^{\circ}$ ), in low northern latitudes, the other in Taurus (galactic long. $100^{\circ}-150^{\circ}$ ) in low southern latitudes. Both run seemingly parallel to each other and perpendicular to the corresponding galactic radius vector. The direction toward the 'centre' of the 'local system' in Carina is also perpendicular to the galactic radius vector of the sun (between both regions of obscurations); it corresponds to the minimum of cosmic absorption.

There are several other well-attested examples of heavy obscurations in the galactic belt; some authorities even admit that the major bifurcation of the Milky Way represents an apparent effect of very distant dark nebulæ. Now having in view the above-mentioned difficulties connected with the existence of individual galactic clouds, it seems natural to make one step further and to reach a new rather radical conclusion. If such an important 